Rend. Lincei Mat. Appl. 19 (2008), 135-140

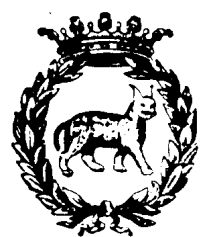

Algebra. - On a theorem of Schmid, by FRAnCESCO ESPOSITO and ANDREA MAFFEI, communicated on 14 December 2007.

ABSTRACT. - We establish for which parabolic subgroups $P$ of a simply connected and semisimple algebraic group $G$ with unipotent radical $U$ and Levi factor $H$ the rings $\mathbb{k}[G / H]^{U}$ and $\mathbb{k}\left[U^{-}\right]$are isomorphic as $H$ algebras. We show a relation of this problem with a theorem of Schmid and we compare the multiplications in the rings $\mathbb{k}\left[U^{-}\right]$and $\mathbb{k}[G / H]$.

KEY WORDS: Semisimple algebraic groups; homogeneous spaces; symmetric spaces; regular functions.

Mathematics Subject Classification (2000): 22E46, $20 \mathrm{G} 05$.

Let $G$ be a simply connected and semisimple algebraic group over an algebraically closed field $\mathbb{k}$. Let $P$ be a parabolic subgroup of $G, U$ its unipotent radical and $H$ a Levi factor. Let also $U^{-}$be the unipotent radical of a parabolic opposite to $P$.

We want to describe the relation between the coordinate rings of $G / H$ and $U^{-}$. We notice that since $U^{-} \cap H=\{1\}$ the inclusion of $U^{-}$in $G$ induces an $H$-equivariant immersion $\imath: U^{-} \hookrightarrow G / H$. Moreover, since every orbit of a unipotent group acting on an affine variety is closed, $l$ is a closed immersion. So we have a surjective morphism of $H$-algebras $\iota^{*}: \mathbb{k}[G / H] \rightarrow \mathbb{k}\left[U^{-}\right]$. Let $\varphi: \mathbb{k}[G / H]^{U} \rightarrow \mathbb{k}\left[U^{-}\right]$be the restriction of $\imath^{*}$. Then our main result is the following

THEOREM. Let $G$ be simple and $\mathbb{k}$ of characteristic 0 . The map $\varphi$ is an isomorphism if and only if either $U$ is commutative, or $G$ is of type $\mathrm{F}_{4}$ and $P$ is the maximal parabolic with semisimple Levi of type $\mathrm{C}_{3}$, or $G$ is of type $B_{n}$ and $P$ is the maximal parabolic with semisimple Levi of type $\mathrm{A}_{n-1}$.

The paper is organized as follows. In Section 1 we study the case of $U$ commutative, in which we can give a simpler proof which partly holds in positive characteristic. This case is related to symmetric varieties. Indeed, for every such $U$, the Levi $H$ is the subgroup of points fixed by an involution of $G$. In particular, we can use the theorem above to show that to determine the decomposition of $\mathbb{k}\left[U^{-}\right]$into $H$-modules is equivalent to determining the decomposition of $\mathbb{k}[G / H]$ into $G$-modules. This relates a theorem of Schmid [5] to a theorem of Helgason [3]. In the recent years there has been some interest in the products of irreducible modules in these two rings [1, 4, 2] and we compare these two products. In the second section we prove the theorem in the general case.

\title{
1. THE SYMMETRIC CASE
}

We keep the notation introduced above. Let also $T \subset H$ be a maximal torus, $T \subset B_{G} \subset P$ a Borel subgroup and $B_{G}^{-}$the opposite Borel. Notice that $H$ acts on $U^{-}$by conjugation. 
Notice that if $V$ is a representation of $G$ then the set $V^{U}$ of points fixed by $U$ is $H$ stable, in particular $\mathbb{k}[G / H]^{U}$ is an $H$-algebra and $\varphi$ is a morphism of $H$-algebras. Notice also that since the image of $U \times U^{-}$is dense in $G / H$ the morphism $\varphi$ is certainly injective.

Let also $U_{G}$ be the unipotent radical of $B_{G}$ and notice that $B_{H}:=B_{G} \cap H$ is a Borel of $H$ and that $U_{H}=U_{G} \cap H$ is the unipotent radical of $B_{H}$.

Finally, we will denote the Lie algebra of a group by the corresponding gothic letter.

THEOREM 1. Assume that the Lie algebra $\mathfrak{g}$ of $G$ admits an invariant nondegenerate bilinear form and that $U$ is commutative. Then:

(i) $\varphi: \mathbb{k}[G / H]^{U} \rightarrow \mathbb{k}\left[U^{-}\right]$is an isomorphism of $H$-algebras;

(ii) the restriction of $\mathrm{l}$ to the $U_{G}$-invariants induces an isomorphism of algebras $\psi$ : $\mathbb{k}[G / H]^{U_{G}} \rightarrow \mathbb{k}\left[U^{-}\right]^{U_{H}}$.

ProOF. (i) We have already noticed that $\varphi$ is injective; we now prove that it is surjective. Notice that $\mathfrak{g}=\mathfrak{u} \oplus \mathfrak{z} \oplus \mathfrak{h}^{\prime} \oplus \mathfrak{u}^{-}$where $\mathfrak{u}$ is the Lie algebra of $U, \mathfrak{u}^{-}$of $U^{-}$, and $\mathfrak{z}$ and $\mathfrak{h}^{\prime}$ are respectively the center and commutator of the Lie algebra of $H$. Let $z \in \mathfrak{z}$ be an element whose centralizer is equal to $H$. Since $z$ is semisimple we have a closed immersion $J$ of $G / H$ in $\mathfrak{g}$. Hence we have the following surjective morphisms of rings:

$$
S\left[\mathfrak{g}^{*}\right] \rightarrow \mathbb{k}[G / H] \rightarrow \mathbb{k}\left[U^{-}\right] .
$$

Notice that $\jmath_{l}\left(U^{-}\right)=U^{-} \cdot z$ is contained in $z \oplus \mathfrak{u}^{-}$. Let now $\mathfrak{g}^{*}=\mathfrak{u}^{*} \oplus \mathfrak{z}^{*} \oplus\left(\mathfrak{h}^{\prime}\right)^{*} \oplus\left(\mathfrak{u}^{-}\right)^{*}$ be the decomposition $\mathfrak{g}^{*}$ induced by the decomposition $\mathfrak{g}=\mathfrak{u} \oplus \mathfrak{z} \oplus \mathfrak{h}^{\prime} \oplus \mathfrak{u}^{-}$. Notice that $J \imath\left(U^{-}\right)=U^{-} \cdot z$ is contained in $z \oplus \mathfrak{u}^{-}$. Hence $\left(\mathfrak{h}^{\prime}\right)^{*}$ and $\mathfrak{u}^{*}$ vanish on $z \oplus \mathfrak{u}^{-}$and $\mathfrak{z}^{*}$ gives constant functions. So we have a surjective map from $S\left[\left(\mathfrak{u}^{-}\right)^{*}\right]$ to $\mathbb{k}\left[U^{-}\right]$. Notice now that since $\mathfrak{h}^{\prime} \oplus \mathfrak{z} \oplus \mathfrak{u}$ is a $U$-stable subspace, so is $\left(\mathfrak{u}^{-}\right)^{*}$, and the restriction map from $S\left(\mathfrak{g}^{*}\right)$ to $S\left[\left(\mathfrak{u}^{-}\right)^{*}\right]$ is $U$-equivariant. Finally, notice that $U^{-}$commutative implies that the action of $U$ on $\mathfrak{u}$ is trivial, and using the invariant form we see that this implies that the action of $U$ on $\left(\mathfrak{u}^{-}\right)^{*}$ is trivial. Hence any function on $U^{-}$can be lifted to a $U$-invariant function on $\mathfrak{g}$, hence also on $G / H$.

(ii) follows immediately from (i).

In Theorem 2 we will characterize all the possible $H$ such that $\varphi$ is an isomorphism in the case of characteristic zero.

From now on we assume the characteristic to be zero.

Let us introduce a little bit of notation. Let $\Lambda=\operatorname{Hom}\left(T, \mathbb{k}^{*}\right)$ be the set of weights of $T$ and let $\Lambda_{H}^{+}$be the subset of weights dominant with respect to $B_{H}$, and $\Lambda_{G}^{+}$those dominant with respect to $B_{G}$. For each $\lambda \in \Lambda_{G}^{+}$(resp. $\Lambda_{H}^{+}$) let $V_{\lambda}$ (resp. $W_{\lambda}$ ) be the irreducible representation of $G$ (resp. $H$ ) of highest weight $\lambda$; notice that $\Lambda_{G}^{+} \subset \Lambda_{H}^{+}$and $V_{\lambda}^{U} \simeq W_{\lambda}$ as $H$-modules for each $\lambda \in \Lambda_{G}^{+}$.

For each $\lambda \in \Lambda_{G}^{+}$let $m_{\lambda}=\operatorname{dim}\left(V_{\lambda}^{*}\right)^{H}$ and let $\Omega$ be the set of dominant weights such that $m_{\lambda}>0$. So from $\mathbb{k}[G] \simeq V \otimes V^{*}$ we obtain $\mathbb{k}[G / H] \simeq \bigoplus V_{\lambda} \otimes \mathbb{k}^{m_{\lambda}}$.

Before studying the general situation we want to explain the relation between Theorem 1 and a theorem of Schmid. We want to characterize first the cases in which $U$ is commutative. We fix some notations. Let $\Phi$ be the root system of $\mathfrak{g}$ with respect to the torus $T$, and $\Delta$ the simple roots corresponding to the choice of the Borel $B$. Moreover, for all $\alpha \in \Phi$ let $x_{\alpha}$ be a root vector of weight $\alpha$. Let also $\Psi \subset \Phi$ be the root system of $H$. 
LEMMA 2. Assume that $G$ is simple. Then the following conditions are equivalent:

(i) $U$ is commutative;

(ii) $\mathfrak{u}$ is irreducible as an $H$-module;

(iii) $P$ is the maximal parabolic associated to a root $\alpha$ which appears with multiplicity 1 in the highest root;

(iv) there is an involution $\sigma$ such that $H=G^{\sigma}$.

ProOF. (ii) $\Rightarrow$ (i). Indeed, $[\mathfrak{u}, \mathfrak{u}]$ is an $H$-submodule of $\mathfrak{u}$ different from $\mathfrak{u}$.

(i) $\Rightarrow$ (iii). Assume first that $P$ is not maximal and let $\alpha, \beta \in \Delta \backslash \Psi$ be distinct simple roots. Since $G$ is simple, the Dynkin diagram is connected; let $\gamma_{1}, \ldots, \gamma_{n}$ with $\gamma_{1}=\alpha$ and $\gamma_{n}=\beta$ be a path in the Dynkin diagram from $\alpha$ to $\beta$. Let $\gamma=\gamma_{1}+\cdots+\gamma_{n-1}$. Then $x_{\gamma}, x_{\beta} \in \mathfrak{u}$ and $\left[x_{\gamma}, x_{\beta}\right] \neq 0$. Let $P$ be the maximal parabolic corresponding to the simple root $\alpha$ and assume that $\alpha$ appears with multiplicity greater than or equal to 2 in the highest root. Then there is a root $\beta$ such that $\alpha$ appears with multiplicity 1 in $\beta$ and $\beta+\alpha \in \Phi$. So $x_{\beta}, x_{\alpha} \in \mathfrak{u}$ and $\left[x_{\alpha}, x_{\beta}\right] \neq 0$.

(iii) $\Rightarrow$ (i). We prove that $x_{\alpha}$ generates $\mathfrak{u}$ as an $H$-module so $\mathfrak{u}$ is an irreducible $H$ module of lowest weight $\alpha$. Let $x_{\beta} \in \mathfrak{u}$. Then $\beta=\alpha+\sum_{i=1}^{n} \gamma_{i}$ with $\gamma_{i} \in \Delta \backslash \Psi$. We proceed by induction on $n$. If $\kappa$ is the Killing form then $\kappa(\beta, \beta)>0$ so either $\kappa(\beta, \alpha)>0$ or $\kappa\left(\beta, \gamma_{i}\right)>0$ for some $i$. In the first case $\gamma=\beta-\alpha \in \Psi$ so $x_{\gamma} \in \mathfrak{h}$ and $x_{\beta}$ is a scalar multiple of $\left[x_{\gamma}, x_{\alpha}\right]$. In the second case $x_{\beta-\gamma_{i}} \in \mathfrak{u}$ and we conclude by induction.

(iii) $\Rightarrow$ (iv). We can define an involution $\sigma$ acting trivially on the root vectors $x_{\beta}$ and $x_{-\beta}$ for $\beta \in \Delta \backslash\{\alpha\}$ and acting as -1 on the root vectors $e_{\alpha}$ and $e_{-\alpha}$.

(iv) $\Rightarrow$ (i). Let $H=G^{\sigma}$. Then $\sigma$ fixes the torus $T$ and the roots $\Phi$. So the action is given by multiplication by -1 on $x_{\beta}$ for $\beta \in \Phi \backslash \Psi$ and by multiplication by 1 on $x_{\beta}$ for $\beta \in \Psi$. Now we can argue as in (i) $\Rightarrow$ (iii).

It follows immediately that for $G$ semisimple, $U$ is commutative if and only if $H$ is the subgroup of points fixed by an involution. In particular, $B_{G}$ has an open orbit in $G / H$ in this case, in particular $m_{\lambda} \leq 1$ for all $\lambda$. Hence if $U$ is commutative we have

$$
\mathbb{k}[G / H]=\bigoplus_{\lambda \in \Omega} V_{\lambda}
$$

and from Theorem 1 by taking $U$-invariants we get

$$
\mathbb{k}\left[U^{-}\right]=\bigoplus_{\lambda \in \Omega} W_{\lambda} .
$$

In particular, using Helgason's description [3] of spherical representations (irreducible representations of $G$ which have a nonzero vector fixed by $H$ ) we see how we can deduce the description of $\mathbb{k}[U]$ as an $H$-module given by Schmid [5] for hermitian symmetric varieties.

REMARK 3. We want to compare the product of two $G$-submodules of $\mathbb{k}[G / H]$ under the usual multiplication in the ring with the product of $H$-submodules of $\mathbb{k}\left[U^{-}\right]$in the case where $U$ is commutative. Recently these products have been studied: in [4, 1] for the case of $G$-submodules of $\mathbb{k}[G / H]$ and in [2] for the case of $H$-submodules of $\mathbb{k}\left[U^{-}\right]$. 
Given $\lambda, \mu \in \Omega$ let $\mathcal{V}(\lambda, \mu)=\left\{v \in \Omega: V_{\nu} \subset V_{\lambda} \cdot V_{\mu} \subset \mathbb{k}[G / H]\right\}$ and similarly define $\mathcal{W}(\lambda, \mu)=\left\{v \in \Omega: W_{\nu} \subset W_{\lambda} \cdot W_{\mu} \subset \mathbb{k}\left[U^{-}\right]\right\}$.

From the previous constructions we obtain $\mathcal{W}(\lambda, \mu) \subset \mathcal{V}(\lambda, \mu)$. We now give an example where equality does not hold.

Let $X_{1}=X_{2}=\mathbb{k}^{n}$ and $X=X_{1} \oplus X_{2}$. Let $G=\operatorname{SL}(X)$ and let $H$ be the Levi subgroup which stabilizes $X_{1}$ and $X_{2}$. The center of $H$ is the one-parameter subgroup $\gamma(t)=t \operatorname{id}_{X_{1}} \oplus t^{-1} \mathrm{id}_{X_{2}}$ and its semisimple part is $\operatorname{SL}\left(X_{1}\right) \times \operatorname{SL}\left(X_{2}\right)$. Let also $V=\mathfrak{s l}(X)$ be the representation of highest weight $\theta$, the highest root of $G$, and notice that $W=V^{U}=$ $X_{1} \otimes X_{2}^{*}$ and that $\gamma(t)$ acts by multiplication by $t^{2}$ on $W$ and so by multiplication by $t^{4}$ on $W \cdot W$. In particular, $0 \notin \mathcal{W}(\theta, \theta)$, while it is easy to see that $0 \in \mathcal{V}(\theta, \theta)$ (see for example [1]).

\section{The GENERAL CASE}

We now go back to the general situation and we classify all $H$ such that the map $\varphi$ introduced in the first section is an isomorphism.

LEMMA 4. Assume that $G$ is simple. If $\varphi: \mathbb{k}[G / H]^{U} \rightarrow \mathbb{k}\left[U^{-}\right]$is an isomorphism then either $\mathfrak{u}$ is irreducible or $G$ is not simply laced and $\mathfrak{u} \simeq W_{\theta} \oplus W_{\theta^{\prime}}$ as $H$-modules where $\theta$ is the highest root of $\mathfrak{g}$ and $\theta^{\prime}$ is the highest short root.

ProOF. From $\mathbb{k}[G / H]^{U} \simeq \mathbb{k}\left[U^{-}\right]$as $H$-modules we deduce that

$$
\mathbb{k}\left[U^{-}\right] \simeq \bigoplus_{\lambda \in \Lambda_{G}^{+}} W_{\lambda} \otimes \mathbb{k}^{m_{\lambda}}
$$

in particular, all the $H$-modules which appear in $\mathbb{k}\left[U^{-}\right]$have a highest weight which is dominant with respect to $B_{G}$.

Notice also that the exponential map gives an algebraic $H$-equivariant isomorphism between $\mathfrak{u}^{-}$and $U^{-}$and in particular $\mathbb{k}\left[U^{-}\right] \simeq \mathbb{k}\left[\mathfrak{u}^{-}\right] \simeq S(\mathfrak{u})$ as $H$-algebras. In particular, the representation $\mathfrak{u}=S^{1}(\mathfrak{u})$ must appear in the sum $\bigoplus_{\lambda \in \Lambda_{G}^{+}} W_{\lambda} \otimes \mathbb{k}^{m_{\lambda}}$. But $\mathfrak{u} \subset \mathfrak{u}_{G}$, the Lie algebra of $U_{G}$ whose weights are given by positive roots. Now if $G$ is simply laced there is only one such root which is dominant with respect to $B_{G}$ and that is the highest root. So in this case $\mathfrak{u}=W_{\theta}$ and it is irreducible. On the other hand, if $G$ is not simply laced there can be two such roots: $\theta$ and $\theta^{\prime}$, and $\mathfrak{u}$ can be either irreducible or isomorphic to $W_{\theta} \oplus W_{\theta^{\prime}}$.

When $\mathfrak{u}$ is an irreducible $H$-module, we are back in the commutative case, so we will have to analyze the other case.

LEMMA 5. Assume that $G$ is a simple Lie group not simply laced. If $\mathfrak{u} \simeq W_{\theta} \oplus W_{\theta^{\prime}}$ as $H$-modules then $P$ is a maximal parabolic corresponding to a simple root $\alpha$ which appears with multiplicity 2 in the highest root and with multiplicity 1 in $\theta^{\prime}$.

Proof. Assume first $P$ is not maximal and consider simple roots $\alpha, \beta$ which are not in the root system of $H$. Let $\mathfrak{u}_{1}$ be the span of the root spaces $\mathfrak{g}_{\gamma}$ with $\gamma$ a positive root where 
$\alpha$ appears with multiplicity 1 and $\beta$ with multiplicity 0 ; similarly, let $\mathfrak{u}_{2}$ be the span of the root spaces $\mathfrak{g}_{\gamma}$ with $\gamma$ a positive root where $\beta$ appears with multiplicity 1 and $\alpha$ with multiplicity 0; finally, let $\mathfrak{u}_{3}$ be the span of the root spaces $\mathfrak{g}_{\gamma}$ with $\gamma$ a positive root where both $\alpha$ and $\beta$ appear with multiplicity 1 . These spaces are not zero and $H$-stable, contrary to the fact that $\mathfrak{u}$ is the sum of two irreducible $H$-modules. Similarly one can treat the case in which $P$ is the maximal parabolic corresponding to a root $\alpha$ which appears with multiplicity at least 3 in the highest root or with multiplicity 2 in both $\theta$ and $\theta^{\prime}$ (this last case actually cannot occur under our hypothesis).

Proposition 6. Assume that $G$ is a simple Lie group not simply laced and that $P$ is a maximal parabolic corresponding to a simple root $\alpha$ which appears with multiplicity 2 in the highest root and with multiplicity 1 in $\theta^{\prime}$ and that $\mathfrak{u} \simeq W_{\theta} \oplus W_{\theta^{\prime}}$. Then $\varphi$ is an isomorphism if and only if $m_{\theta^{\prime}}=1$ and $W_{\theta}$ is not an irreducible factor of $S^{2}\left(W_{\theta^{\prime}}\right)$.

Proof. We notice first that $\left(V_{\theta}^{*}\right)^{H}=\mathfrak{g}^{H}=Z(\mathfrak{h})=\mathfrak{z}$, the center of the Lie algebra of $H$, is one-dimensional and spanned by the fundamental coweight $z=\omega_{\alpha}^{\vee}$ relative to the root $\alpha$. In particular, $m_{\theta}=1$.

Notice also that $z$ acts as $\langle\lambda, z\rangle$ times the identity on the representation of $W_{\lambda}$. In particular, it acts as the identity on $W_{\theta^{\prime}}$ and twice the identity on $W_{\theta}$. So, since $S^{1}(\mathfrak{u})=$ $W_{\theta} \oplus W_{\theta^{\prime}}$ generates $S(\mathfrak{u})$ and the multiplication is equivariant, if we look at the action of $z$ on $S(\mathfrak{u})$ we see that $W_{\theta^{\prime}}$ can appear only in $S^{1}(\mathfrak{u})$ and $W_{\theta}$ can appear only as a factor of $S^{1}(\mathfrak{u})$ and in $S^{2}(\mathfrak{u})$ as a factor of $S^{2}\left(W_{\theta^{\prime}}\right)$.

In particular, if $W_{\theta}$ is not an irreducible factor of $S^{2}\left(W_{\theta^{\prime}}\right)$ then $W_{\theta}$ and $W_{\theta^{\prime}}$ appear with multiplicity one in $S(\mathfrak{u})$ and their sum is equal to $\mathfrak{u}=S^{1}(\mathfrak{u})$. Moreover, if $m_{\theta^{\prime}}=1$ then $W_{\theta}$ and $W_{\theta^{\prime}}$ also appear with multiplicity 1 in $\mathbb{k}[G / H]^{U}$. So since $\varphi$ is always injective its image contains $S^{1}(\mathfrak{u})$ which generates $S(\mathfrak{u})$, hence it is surjective.

Conversely, assume that $\varphi$ is an isomorphism. Then $S[\mathfrak{u}] \simeq \bigoplus_{\lambda \in \Omega} W_{\lambda} \otimes \mathbb{k}^{m_{\lambda}}$. We have already noticed that $m_{\theta}=1$ in this case and $W_{\theta}$ indeed appears in degree one. So it cannot appear also in degree two as a factor of $S^{2}\left(W_{\theta^{\prime}}\right)$, hence $W_{\theta}$ is not an irreducible factor of $S^{2}\left(W_{\theta^{\prime}}\right)$. Also we have already noticed that $W_{\theta^{\prime}}$ can appear only in degree one where it appears with multiplicity 1 , so we must have $m_{\theta^{\prime}}=1$.

THEOREM 7. Let $G$ be simple. Then $\varphi$ is an isomorphism if and only if either $U$ is commutative, or $G$ is of type $\mathrm{F}_{4}$ and $P$ is the maximal parabolic with semisimple Levi of type $\mathrm{C}_{3}$, or $G$ is of type $B_{n}$ and $P$ is the maximal parabolic with semisimple Levi of type $\mathrm{A}_{n-1}$.

ProOF. From Lemma 1 we must analyze the following cases (the simple roots $\alpha_{i}$ and the fundamental weights $\omega_{i}$ are numbered as in Bourbaki):

CASE 1: $\quad G$ of type $\mathrm{B}_{n}$ and $P$ a maximal parabolic corresponding to the $i$-th simple root with $i=2, \ldots, n$. We have $\theta^{\prime}=\sum \alpha_{i}=\omega_{1}$ and $\theta=\alpha_{1}+2 \sum_{i>1} \alpha_{i}=\omega_{2}$. In particular, $V_{\theta^{\prime}}^{H}=0$ if $i \neq n$ and is one-dimensional for $i=n$. Finally, $S^{2}\left(W_{\theta^{\prime}}\right)=S^{2}\left(\mathbb{k}^{n}\right)$ is irreducible in this case and does not contain $W_{\theta}=\Lambda^{2}\left(\mathbb{k}^{n}\right)$ and Proposition 6 applies.

CASE 2: $\quad G$ of type $\mathrm{C}_{n}$ and $P$ a maximal parabolic corresponding to the $n$-th simple root. We have $\theta^{\prime}=\alpha_{1}+2 \sum_{1<i<n} \alpha_{i}+\alpha_{n}=\omega_{2}$ and $\theta=2 \sum_{i<n} \alpha_{i}+\alpha_{n}$. In particular $S^{2}\left(W_{\theta^{\prime}}\right)=S^{2}\left(\Lambda^{2}\left(\mathbb{k}^{2 n}\right) / \mathbb{k}\right) \supset W_{\theta}=S^{2}\left(\mathbb{k}^{2 n}\right)$ and Proposition 6 applies. 
CASE 3: $\quad G$ of type $\mathrm{F}_{4}$ and $P$ a maximal parabolic corresponding to the first simple root. We have $\theta^{\prime}=\alpha_{1}+2 \alpha_{2}+3 \alpha_{3}+2 \alpha_{4}=\omega_{4}$ and $\theta=2 \alpha_{1}+3 \alpha_{2}+4 \alpha_{3}+2 \alpha_{4}=\omega_{1}$. Both conditions of Proposition 6 can be easily checked.

CASE 4: $G$ of type $\mathrm{G}_{2}$ and $P$ a maximal parabolic corresponding to the second simple root. We have $\theta^{\prime}=2 \alpha_{1}+\alpha_{2}=\omega_{1}$ and $\theta=3 \alpha_{1}+2 \alpha_{2}=\omega_{2}$. In this case $W_{\theta^{\prime}}^{H}=0$ and Proposition 6 applies.

As already noticed in the introduction, for a general semisimple $G$ it immediately follows that $\varphi$ is an isomorphism if and only if $G \supset H$ is a product of the cases listed in the Theorem.

\section{REFERENCES}

[1] R. CHIRIVÌ - A. MAFFEI, Projective normality of complete symmetric varieties. Duke Math. J. 122 (2004), 93-123.

[2] T. Enright - N. Wallach, A Pieri rule for Hermitian symmetric pairs. II. Pacific J. Math. 216 (2004), 51-61.

[3] S. Helgason, A duality for symmetric spaces with applications to group representations. Adv. Math. 5 (1970), 1-154.

[4] S. Kannan, Projective normality of the wonderful compactification of semisimple adjoint groups. Math. Z. 239 (2002), 673-682.

[5] W. SCHMID, Die Randwerte holomorpher Funktionen auf hermitesch symmetrischen Räumen. Invent. Math. 9 (1969/1970), 61-80.

Received 20 November 2007,

and in revised form 20 December 2007

Andrea Maffei

Dipartimento di Matematica Università di Roma "La Sapienza" piazzale Aldo Moro 5

00185 Roma, Italy amaffei@mat.uniroma1.it

Francesco Esposito Dipartimento di Matematica Pura e Applicata

Università degli Studi di Padova via Trieste 63

35121 PADOVA, Italy esposito@math.unipd.it 